\title{
Rationale and design of XARENO: XA inhibition in RENal patients with non-valvular atrial fibrillation. Observational registry
}

\author{
Reinhold Kreutz', Gilbert Deray ${ }^{2}$, Jürgen Floege ${ }^{3}$, Marianne Gwechenberger ${ }^{4}$, Kai Hahn ${ }^{5}$, Andreas R Luft ${ }^{6}$, \\ Pontus Persson ${ }^{7}$, Jan Beyer-Westendorf ${ }^{8}$
}

\author{
${ }^{1}$ Charité - Universitätsmedizin Berlin, corporate member of Freie Universität Berlin, Humboldt-Universität zu Berlin, and Berlin Institute of Health, \\ Institute of Clinical Pharmacology and Toxicology, Berlin, Germany \\ ${ }^{2}$ Department of Nephrology, Pitié-Salpêtrière University Hospital, Paris, France \\ ${ }^{3}$ Division of Nephrology and Clinical Immunology, RWTH Aachen University Hospital, Aachen, Germany \\ ${ }^{4}$ University Department of Internal Medicine II, Division of Cardiology, Medical University of Vienna, Vienna, Austria \\ ${ }^{5} \mathrm{~B}$ Braun Medical Care AG Dialysezentrum Hochfelden, Zürich, Switzerland \\ ${ }^{6}$ Department of Neurology, Universitätsspital Zürich, Switzerland and Cereneo Center for Neurology and Rehabilitation, Vitznau, Switzerland \\ ${ }^{7}$ Charité - Universitätsmedizin Berlin, corporate member of Freie Universität Berlin, Humboldt-Universität zu Berlin, and Berlin Institute of Health, \\ Institute of Translational Physiology, Berlin, Germany \\ ${ }^{8}$ Thrombosis Research Unit, Department of Medicine I, Division Haematology, University Hospital Carl Gustav Carus Dresden, Dresden, Germany
}

\author{
Correspondence to: \\ Prof. Reinhold Kreutz, MD, PhD, \\ Institute of Clinical Pharmacology \\ and Toxicology, \\ Charitéplatz 1, 10117 Berlin, \\ Germany, \\ phone: +49 30450525112 , \\ e-mail: \\ reinhold.kreutz@charite.de \\ Copyright by the Author(s), 2021 \\ Kardiol Pol. 2021. \\ 79 (11): 1265-1267 \\ DOI: 10.33963/KP.a2021.0134 \\ Received: \\ September 16, 2021 \\ Revision accepted: \\ October 10, 2021 \\ Published online: \\ October 11, 2021
}

\section{INTRODUCTION}

Non-valvular atrial fibrillation (NVAF) and chronic kidney disease (CKD) are frequently associated with each other [1]. In the pivotal randomized controlled trials (RCTs) that lead to the approval of direct oral anticoagulants (DOACs) for stroke prevention in patients with NVAF, patients with an estimated creatinine clearance $(\mathrm{CrCl})<25-30 \mathrm{ml} / \mathrm{min}$ [1-3] were excluded, and the overall frequency of patients with moderate renal impairment $(\mathrm{CrCl}$ $<50 \mathrm{ml} / \mathrm{min}$ ) was about $19 \%$ [4]. This applies also to the Rivaroxaban Once Daily Oral Direct Factor Xa Inhibition Compared with Vitamin K Antagonism for Prevention of Stroke and Embolism Trial in Atrial Fibrillation (ROCKET AF) [5], in which $21 \%$ of patients had a $\mathrm{CrCl}$ $<50 \mathrm{ml} / \mathrm{min}$. Importantly, among the latter group, all patients who were randomized to rivaroxaban in ROCKET-AF received the lower $15 \mathrm{mg}$ once daily dose $[5,6]$. Based on pharmacokinetic rationales, this reduced dose was also subsequently approved for use in clinical practice in NVAF patients with severe renal impairment $(\mathrm{CrCl}$ range 15 to $30 \mathrm{ml} / \mathrm{min})[1$, $3,7]$. Interestingly, previous studies indicated a potential benefit of anticoagulation with DOACs versus vitamin $\mathrm{K}$ antagonist (VKA) for renal outcomes including a slower decline of renal function over time and reduced development of CKD stage $5[8,9]$. The evaluation of the efficacy and safety of anticoagulation with rivaroxaban as compared to VKA in clinical practice is, therefore, of interest in this vulnerable patient group $[1,3,10]$.

\section{METHODS}

The XARENO study is an investigator-initiated, multicenter, prospective, and non-interventional registry conducted in Germany, Austria, Switzerland, France, Belgium, and Luxembourg. Management of patients is carried out in clinical routine at the discretion of the participating physician. Recruitment of patients is conducted by investigators at selected study centers. The study sponsor obtained approval from an independent Ethics Committee or Institutional Review Board in all participating countries. The XARENO registry is independently managed by the sponsor, GWTTUD (Gesellschaft fürWissens- und Technologitransfer, Technische Universität Dresden, Germany), and a scientific steering committee. Members of the latter are exclusively the authors of this report. The study is registered with the unique identifier: NCT02663076.

Inclusion criteria are NVAF as diagnosed by the participating physicians, adult age 


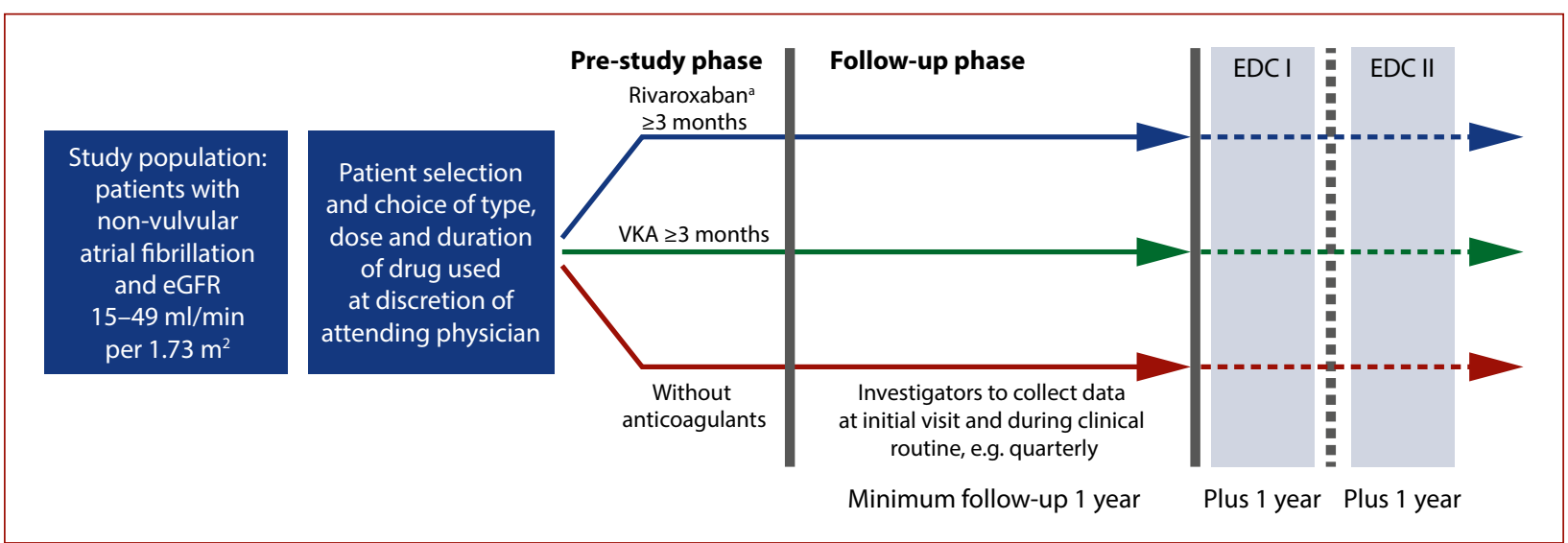

Figure 1. Study design for the XARENO study. During the conduct of the registry, it became apparent that the recruitment of patients was slower and lower with overall approximately $>700$ patients rather than 1000 patients included in the rivaroxaban and VKA group of patients, respectively. Therefore, the study protocol was amended and an extended data collection (EDC) period I, for one year is planned. In addition, depending on the overall number of patients still available in the registry, another extension for one year may be conducted (EDC II)

aThe recommended dose for rivaroxaban is $15 \mathrm{mg}$ once daily, although physicians may, in clinical practice, change the dose at their discretion Abbreviations: eGFR, estimated glomerular filtration rate; VKA, vitamin K antagonist

( $\geq 18$ years), and an estimated glomerular filtration rate (eGFR) between 15 and $49 \mathrm{ml} / \mathrm{min}$ per $1.73 \mathrm{~m}^{2}$. (Supplementary material, Table S1). Patients are either treated with rivaroxaban or VKA while patients without anticoagulation at the discretion of attending physicians are also enrolled for explorative analyses. Patients should have been treated with either the recommended $15 \mathrm{mg}$ once daily dose of rivaroxaban or VKA for the $\geq 3$ previous months before enrolment, at the earliest in January 2012 (when rivaroxaban became available for treatment in NVAF in the participating countries). Thus, patients will continue their previous anticoagulation treatment when included in the XARENO study. Further inclusion criteria are summarized in Supplementary material, Table S1.

Exclusion criteria include chronic treatment with parenteral anticoagulants or DOACs other than rivaroxaban and current or expected renal-replacement therapy (Supplementary material, Table S1).

Pre-specified follow-up is at least 12 months followed by a planned extended data collection period for 1 up to 2 additional years (Figure 1). Primary outcomes for the comparison between rivaroxaban and VKA groups after 12 months include progression of CKD, i.e. the decline in eGFR in $\mathrm{ml} / \mathrm{min}$ per $1.73 \mathrm{~m}^{2}$ and other efficacy and safety outcomes as summarized in Supplementary material, Table S2. eGFR will be estimated by the Chronic Kidney Disease Epidemiology Collaboration (CKD-EPI) equation [11]. In addition, creatinine clearance will be analyzed and estimated with the Cockcroft-Gault formula [12]. Secondary outcomes include other renal events, rates, causes and length of hospitalizations, persistence with oral anticoagulation therapy, and reasons for discontinuation of this treatment (Supplementary material, Table S3). All outcome events will be adjudicated by a central blinded adjudication committee that includes members of the scientific steering committee. Event adjudication will be based on all available blinded source data.

Antiplatelet therapy could be used in patients in the rivaroxaban or VKA groups at the discretion of the attending physicians. The non-interventional design of the XARENO registry records only the type and dosage of antithrombotic therapy at baseline and during follow-up without providing recommendations for treatment choice, switches, or discontinuation of antithrombotic therapy. To allow for meaningful comparisons between rivaroxaban and VKA, planned prospective treatment with apixaban, dabigatran, edoxaban, or any non-approved experimental anticoagulant drug at baseline excluded patients from the study.

Informed consent and documentation of baseline characteristics of all eligible patients are obtained by the attending physician. Data for eGFR and $\mathrm{CrCl}$ (based on serum creatinine measurements), current anticoagulation therapy, concomitant medications, bleeding events, thromboembolic and cardiovascular events, and all further events of interest are collected every three months where available and as obtained during routine medical practice.

The primary statistical analysis (details are presented in suppl. statistical analysis) includes a descriptive analysis of the primary and secondary outcomes. A direct comparison between groups will be performed only between the rivaroxaban and VKA (anticoagulation) groups. The rationale for the sample size for the analysis of the primary endpoint of progression of CKD is based on a previous report [8] and a mean difference in GFR of at least $1 \mathrm{ml} / \mathrm{min}$ per $1.73 \mathrm{~m}^{2}$ after 12 months. All primary and secondary outcome variables will be analyzed in the intention-to-treat analysis (ITT) under the initial treatment (safety population). Patients that switched treatment will be censored after the interval of their initial treatment. These variables will be analyzed using survival analysis and Kaplan-Meier estimation of 
time to the first event by treatment (rivaroxaban vs. VKA). The potential for allocation bias in the XARENO study will be addressed by performing additional propensity score matching to control for the effects of unbalanced covariates [13]. Thus, in addition to ITT, a propensity score matched analysis for the comparison between rivaroxaban and VKA groups will be performed. The aim in the group of patients without anticoagulation is of explorative nature, i.e., it is to collect data on patient characteristics and to describe the natural course of these patients with CKD and NVAF for the same outcome variables analyzed in the two groups with oral anticoagulation therapy.

\section{RESULTS AND DISCUSSION}

The XARENO study aims to provide valuable prospective information on the real-world effectiveness of rivaroxaban versus VKA for the vulnerable group of patients with NVAF and advanced non-dialysis dependent $\operatorname{CKD}[1,3,10]$. This also includes the evaluation of a potential protective effect of rivaroxaban on the progression of CKD possibly due to favorable vascular mechanisms as compared to VKA [14]. In this regard, it will not only complement the RCT evidence from the ROCKET-AF trial with real-world data but also fill a knowledge gap in patients with advanced CKD stage 4, with observational prospective data.

\section{Supplementary material}

Supplementary material is available at https://journals. viamedica.pl/kardiologia_polska.

\section{Article information}

Acknowledgments: The authors are very grateful for the support by K. Jersemann, S. Leupold, and A. Polowinski (from GWT, Dresden, Germany), and by J. Berdougo, E. Delorière, and P. Naidoo from ADITEN (Paris, France), and by K. Sahin (ClinStat, Cologne, Germany).

Funding: Funded by Bayer AG as an investigator-initiated study.

Registration: The XARENO study has been registered on URL: www. clinicaltrials.gov; unique identifier: NCT02663076.

Conflict of interests: RK has received the support of research by Bayer AG and personal honoraria from Bayer AG, Berlin-Chemie Menarini, Daiichi Sankyo, Ferrer, Merck, Sanofi, and Servier. GD has received personal honoraria from Bayer AG. JF has received personal honoraria from Amgen, Bayer AG, Fresenius, and Vifor. MG has received personal honoraria from Boehringer Ingelheim, Bayer AG and Daiichi Sankio. KH has received personal honoraria from Bayer AG, Daiichi Sankyo, Berlin Chemie, Vifor Pharma, Astra Zeneca, and financial support from AMGEN.

ARL has received personal honoraria from Bayer AG, Amgen, and Moleac. He has received research funding from the P\&K Pühringer Foundation, The LOOP Zurich, the University of Zurich, and the Swiss National Science Foundation. PP has received personal honoraria from Bayer AG (regarding renal safety) and has received support for research on contrast media and the kidney by Bayer AG. JBW has received institutional research support and personal honoraria from Bayer AG, Daiichi Sankyo, Pfizer, and Portola/Alexion.

Open access: This article is available in open access under Creative Common Attribution-Non-Commercial-No Derivatives 4.0 International (CC BY-NC-ND 4.0) license, allowing to download articles and share them with others as long as they credit the authors and the publisher, but without permission to change them in any way or use them commercially. For commercial use, please contact the journal office at kardiologiapolska@ptkardio.pl.

How to cite: Kreutz R, Deray G, Floege J, et al. Rationale and design of XARENO: XA inhibition in RENal patients with non-valvular atrial fibrillation. Observational registry. Kardiol Pol. 2021; 79(11): 1265-1267, doi: 10.33963/KP.a2021.0134.

\section{REFERENCES}

1. Kumar S, Lim E, Covic A, et al. Anticoagulation in concomitant chronic kidney disease and atrial fibrillation: JACC review topic of the week. J Am Coll Cardiol. 2019; 74(17): 2204-2215, doi: 10.1016/j.jacc.2019.08.1031, indexed in Pubmed: 31648714.

2. Hindricks G, Potpara T, Dagres N, et al. 2020 ESC Guidelines for the diagnosis and management of atrial fibrillation developed in collaboration with the European Association for Cardio-Thoracic Surgery (EACTS): The Task Force for the diagnosis and management of atrial fibrillation of the European Society of Cardiology (ESC) Developed with the special contribution of the European Heart Rhythm Association (EHRA) of the ESC. Eur Heart J. 2021; 42(5): 373-498, doi: 10.1093/eurheartj/ehaa612, indexed in Pubmed: 32860505.

3. Weir MR, Kreutz R. Influence of renal function on the pharmacokinetics, pharmacodynamics, efficacy, and safety of non-vitamin Kantagonist oral anticoagulants. Mayo Clin Proc. 2018; 93(10): 1503-1519, doi: 10.1016/j. mayocp.2018.06.018, indexed in Pubmed: 30286834.

4. Ruff CT, Giugliano RP, Braunwald E, et al. Comparison of the efficacy and safety of new oral anticoagulants with warfarin in patients with atrial fibrillation: a meta-analysis of randomised trials. Lancet. 2014; 383(9921): 955-962, doi: 10.1016/S0140-6736(13)62343-0, indexed in Pubmed: 24315724.

5. Patel MR, Mahaffey KW, Garg J, et al. ROCKET AF Investigators. Rivaroxaban versus warfarin in nonvalvular atrial fibrillation. N Engl J Med. 2011; 365(10): 883-891, doi: 10.1056/NEJMoa1009638, indexed in Pubmed: 21830957.

6. Fox KAA, Piccini JP, Wojdyla D, et al. Prevention of stroke and systemic embolism with rivaroxaban compared with warfarin in patients with non-valvular atrial fibrillation and moderate renal impairment. Eur Heart J. 2011; 32(19): 2387-2394, doi: 10.1093/eurheartj/ehr342, indexed in Pubmed: 21873708.

7. Steffel J, Collins R, Antz M, et al. External reviewers. 2021 European Heart Rhythm Association practical guide on the use of non-vitamin $\mathrm{K}$ antagonist oral anticoagulants in patients with atrial fibrillation. Europace. 2021; 23(10): 1612-1676, doi: 10.1093/europace/euab065, indexed in Pubmed: 33895845.

8. Böhm M, Ezekowitz MD, Connolly SJ, et al. Changes in renal function in patients with atrial fibrillation: an analysis from the RE-LY trial. J Am Coll Cardiol. 2015; 65(23):2481-2493, doi: 10.1016/j.jacc.2015.03.577, indexed in Pubmed: 26065986.

9. Yao X, Tangri N, Gersh BJ, et al. Renal outcomes in anticoagulated patients with atrial fibrillation. J Am Coll Cardiol. 2017; 70(21): 2621-2632, doi: 10.1016/j.jacc.2017.09.1087, indexed in Pubmed: 29169468.

10. Undas A, Drabik L, Potpara T. Bleeding in anticoagulated patients with atrial fibrillation: practical considerations. Kardiol Pol. 2020; 78(2): 105-116, doi: 10.33963/kp.15205, indexed in Pubmed: 32108755.

11. Levey AS, Stevens LA, Schmid CH, et al. CKD-EPI (Chronic Kidney Disease Epidemiology Collaboration). A new equation to estimate glomerular filtration rate. Ann Intern Med. 2009; 150(9):604-612, doi: 10.7326/00034819-150-9-200905050-00006, indexed in Pubmed: 19414839.

12. Cockcroft DW, Gault MH. Prediction of creatinine clearance from serum creatinine. Nephron. 1976; 16(1):31-41, doi: 10.1159/000180580, indexed in Pubmed: 1244564.

13. Collins GS, Le Manach Y.Comparing treatment effects between propensity scores and randomized controlled trials: improving conduct and reporting. Eur Heart J. 2012; 33(15): 1867-1869, doi: 10.1093/eurheartj/ehs186, indexed in Pubmed: 22745354.

14. Kreutz R, Camm AJ, Rossing P. Concomitant diabetes with atrial fibrillation and anticoagulation management considerations. Eur Heart J Suppl. 2020; 22(Suppl 0): 078-086, doi: 10.1093/eurheartj/suaa182, indexed in Pubmed: 33380946. 